\title{
Chromosome damage in human cells induced by UMTS mobile telephony radiation
}

\author{
Dimitris J. Panagopoulos ${ }^{1,2,3}$ \\ ${ }^{1}$ National Center for Scientific Research "Demokritos", Athens, Greece \\ ${ }^{2}$ Choremeion Research Laboratory, Medical School, National and Kapodistrian University of Athens, Greece \\ ${ }^{3}$ EMF-Biophysics Research Laboratory, Athens, Greece
}

\begin{abstract}
Environmental exposure to modern microwave telecommunication electromagnetic fields (EMFs) has increased to unprecedented levels with consequent health complaints and concerns. Many studies have already reported genotoxic effects on a variety of organisms and cell/tissue types. Human peripheral blood lymphocytes from six healthy donors were stimulated for mitosis and exposed to microwave EMF of Universal Mobile Telecommunications System (UMTS) or third generation (3G) Mobile Telephony (MT) EMF/radiation emitted by a commercially available mobile phone handset. Lymphocytes exposed during the $\mathrm{G}_{2}$ phase of the cell division cycle and observed at metaphase, exhibited chromatid-type aberrations (gaps and breaks) at highly significant percentages - up to $275 \%$ - compared to the control (sham-exposed) samples. Each subject exhibited a different sensitivity to the microwave exposure. Moreover, the percentages of aberrations in the control samples among subjects were different due to genetic and environmental factors. The MT EMF exposure induced mainly achromatic lesions (gaps), and secondarily terminal deletions (breaks) in a smaller degree. In conclusion, the present study shows that microwave 3G MT EMF/radiation - within the current exposure limits - has significant genotoxic action on human cells, and human exposure to this EMF/radiation should be kept at levels as low as possible.
\end{abstract}

Key words: Electromagnetic fields - Mobile phones - Lymphocytes - Chromosome aberrations - DNA damage

\section{Introduction}

Increasing concern is raised during the past few decades among the scientific community and the general public, regarding the potential adverse effects of man-made electromagnetic fields (EMFs) and more specifically radio-frequency (RF)/microwave EMFs/radiation on human health. The International Agency for Research on Cancer (IARC) under the weight of the accumulating scientific evidence, classified $\mathrm{RF} /$ microwave radiation as possibly carcinogenic to humans (IARC 2013). Extremely low frequency (ELF)

Correspondence to: Dimitris Panagopoulos, Laboratory of Health Physics, Radiobiology and Cytogenetics, Institute of Nuclear and Radiological Sciences and Technology, Energy and Safety. National Center for Scientific Research “Demokritos”, 60037 Ag. Paraskevi, Athens, Greece

E-mail: dpanagop@biol.uoa.gr
EMFs (up to $3000 \mathrm{~Hz}$ ) was already classified by IARC as possibly carcinogenic as well (IARC 2000). Modern microwave radiation applied in telecommunications and other applications always combines RF carrier waves with ELF pulsing and modulation in order to increase the amount and speed of transmitted information (speech, text, images, video, Internet, etc.) (Panagopoulos 2011, 2017, 2019).

Previous experiments of my group had shown intense bioactivity of Global System for Mobile telecommunications (GSM) or second generation (2G) mobile telephony (MT) EMF exposure. More specifically a 6-min daily exposure for a few days was shown to induce DNA damage in fruit fly ovarian cells to up to $60 \%$ of the number of exposed egg chambers (Panagopoulos et al. 2007, 2010).

Universal Mobile Telecommunications System (UMTS) or third generation (3G) MT EMFs/radiation emitted by commercially available mobile phone handsets and base antennas is today a most usual type of modern microwave 
telecommunications radiation exposing daily billions of mobile phone users throughout the world. While 2G (GSM) is still in use and $4 \mathrm{G}$ is the newest type with a higher carrier frequency and mobile broadband Internet access, telecommunication industry is already preparing the installation of the 5G MT system with an even higher carrier frequency and denser network of base antennas in order to provide even faster data transmission (Singh et al. 2017).

Many studies have reported genotoxic effects of RF/ microwave and especially MT EMFs on a variety of organisms and cell/tissue types (Panagopoulos 2019). In a recent study of the USA National Toxicology Program (NTP) on carcinogenicity, rats were exposed for 2 years, $9 \mathrm{~h}$ per day, in a simulated near-field of a mobile phone antenna emitting $2 \mathrm{G}$ or $3 \mathrm{G}$ MT EMFs. Exposed rats developed brain and heart cancer for both lower $(1.5 \mathrm{~W} / \mathrm{kg})$ and higher $(3$ and $6 \mathrm{~W} / \mathrm{kg}$ ) specific absorption rate (SAR) levels than the current exposure limit $(2 \mathrm{~W} / \mathrm{kg})$ for the human head (NTP 2018; ICNIRP 1998). An Italian life-span exposure study of rats in a simulated GSM 1800 far-field, also found induction of heart schwannomas and brain glial tumors, in agreement with the results of the NTP study (Falcioni et al. 2018). In another recent study comparing the bioactivity between $2 \mathrm{G}$ and $3 \mathrm{G}$ MT EMFs/radiation emitted by an active mobile phone, both types of MT EMFs were found to induce DNA damage on the developing liver of chick embryos, with $3 \mathrm{G}$ (UMTS) being even more genotoxic/bioactive than $2 \mathrm{G}$ (GSM) (D'Silva et al. 2017).

Human peripheral blood lymphocytes - naturally arrested in the $G_{0}$ phase and stimulated for cell division (mitosis) - are a well-known model for the assessment of genotoxicity of environmental agents such as ionizing radiation, smoking, pharmaceuticals, etc. (IAEA 2011). One of the most sensitive assays to assess human sensitivity to low levels of ionizing radiation is the $G_{2}$ assay, which is considered to allow observation of unrepaired DNA lesions converted into chromosomal damage during transition from $\mathrm{G}_{2}$ to M-phase (Terzoudi and Pantelias 2006; Pantelias and Terzoudi 2010, 2011; Terzoudi et al. 2011).

Experiments searching the effects of MT EMFs exposure on human peripheral blood lymphocytes have also been conducted. Belyaev et al. $(2005,2009)$ found that GSM-like or UMTS-like EMF affects chromatin conformation (a sign of cytotoxic action). Schwarz et al. (2008) reported no induction of genotoxic effects in human lymphocytes by UMTSlike exposure, while the same exposure induced genotoxic effects in human fibroblasts. El-Abd and Eltoweissy (2012) found that MT EMF at UMTS carrier frequency 1950 $\mathrm{MHz}$ and $\mathrm{SAR}=2 \mathrm{~W} / \mathrm{kg}$ for exposure durations 5-30 min induced DNA strand breaks and chromosomal aberrations. The damage increased with increasing exposure duration, and was attributed to oxidative stress induced by the EMF exposure. Other studies have reported contradicting or confusing results, such as a genotoxic action depending on carrier frequency (Markova et al. 2005), no effect or even beneficial effect (adaptive response) when combined with genotoxic chemicals (Zeni et al. 2003, 2012), intensifying or diminishing the genotoxic effect of chemicals or ultraviolet radiation (Baohong et al. 2005, 2007), and no effect or intensifying the genetic damage caused by $\mathrm{x}$-rays (Manti et al. 2008).

Unfortunately, all the above in vitro studies on human peripheral blood lymphocytes, employed simulated MT EMFs emitted by generators which are very different than the real MT EMFs emitted by commercially available mobile phones and base antennas (Panagopoulos 2017, 2019), and thus the relevance of these studies in real-life is very poor. Two recent studies examined peripheral blood lymphocytes from people residing in the vicinity of MT base stations and thus exposed to real-life MT EMFs/radiation emitted by the base antennas. Both studies found significantly increased genetic damage compared to control groups residing more than $300 \mathrm{~m}$ away from the antennas/cell towers (Gulati et al. 2016; Zothansiama et al. 2017).

The purpose of the present study was to evaluate the potential genotoxicity of UMTS (3G) MT EMFs/radiation emitted by a commercially available mobile phone handset on human cells. An additional purpose was to establish a reliable method to evaluate EMF-genotoxicity in humans, and test the $G_{2}$ assay as such. Although similar protocols have been applied before (Garaj-Vrhovac and Orescanin 2009), no identical assay has been employed to assess sensitivity to EMFs/non-ionizing radiation, especially in combination with a real-life MT EMF-exposure, and therefore the present study is novel.

\section{Materials and Methods}

\section{Blood culture and lymphocyte preparation}

After obtaining consent, blood samples were collected from 6 healthy non-smoker adult individuals (one sample from one individual in each experiment) in glass tubes with heparin, for analysis of chromosomal sensitivity to mobile phone exposure. The subjects were both males and females, 28-42 years old, with "moderate" mobile phone use (no more than $\sim 30$ min total daily conversation on their mobile phones), and no reported history of major illnesses or any regular medication. Apart from this, no specific differences between the subjects were searched, since each subject had its own control sample. Whole blood samples were cultured in RPMI 1640 medium (Biochrom AG, Germany), supplemented with $10 \%$ fetal bovine serum (FBS), 1\% L-glutamine (2 $\mathrm{mM}$ ), antibiotics (penicillin: $100 \mathrm{U} / \mathrm{ml}$; streptomycin: $100 \mu \mathrm{g} / \mathrm{ml}$ ), and $2 \%$ phytohaemagglutinin (PHA). PHA 
was dissolved in water at a concentration of $0.24 \mathrm{mg} / \mathrm{ml}$. For culturing and collecting the lymphocytes and for the application of the $G_{2}$-assay, the standard protocols were followed (Pantelias and Terzoudi 2011).

As already mentioned, lymphocytes are normally arrested in the $G_{0}$ phase. PHA was used to stimulate the lymphocytes to enter the mitotic cycle. For each subject, a single culture was prepared in a $200 \mathrm{ml}$ flask (which was later divided into individual samples/groups) to ensure identical culture conditions and treatment for all individual samples/groups in each experiment. The culture was incubated for $72 \mathrm{~h}$, at $37^{\circ} \mathrm{C}$ in a humidified incubator with an atmospheric content of $5 \% \mathrm{CO}_{2}$ and $95 \%$ air.

The lymphocyte mitotic cycle duration is about $24 \mathrm{~h}$. Following the standard $\mathrm{G}_{2}$ protocol, we incubated the culture for three days $(72 \mathrm{~h})$, in order to collect more cells at the stage of metaphase during mitosis (Pantelias and Terzoudi 2011; IAEA 2011). After $72 \mathrm{~h}$ of incubation the single blood culture was subdivided into individual samples/groups in identical $30 \mathrm{ml}$ rectangular plastic flasks. [Each individual group contained: $0.5 \mathrm{ml}$ blood, $5 \mathrm{ml}$ culture medium, $100 \mu \mathrm{l}$ $\mathrm{PHA}$. After the separation of the initial culture into individual groups/samples, blood samples were either exposed to the UMTS MT EMF ("exposed samples") in another room of the laboratory (called "exposure room") as described in section "EMF exposure procedure", or simply transferred for the same time (sham-exposed) to the exposure room ("control samples"). [Additional individual samples were used for additional tests which will be described in future publications].

After exposures/sham-exposures were completed ( $30 \mathrm{~min}$ after the beginning of the exposure procedure) and both the exposed and the control samples were returned back to the room with the culture chamber (called "culture room"), individual groups/samples were treated with colcemid (50 $\mu$ l added to each individual group) for $60 \mathrm{~min}$, to arrest dividing cells at metaphase. Colcemid is a known reagent that prohibits the formation of attractus in the dividing cells, and thus prohibiting the cells to proceed from the metaphase to the anaphase. Keeping the cells in metaphase makes their condensed chromosomes clearly observable by light microscopy for possible aberrations. The period of the colcemid treatment $(60 \mathrm{~min})$ right after the termination of exposure/sham-exposure plus the exposure/sham-exposure period $(\sim 1.5 \mathrm{~h}$ in total) determines the phases of the celldivision cycle that were exposed and then collected in metaphase. In this case, the $1.5 \mathrm{~h}$-period determines that the cells collected for observation were at the late $\mathrm{G}_{2}$ or early mitotic (prophase) stages during the exposure/sham-exposure. Cells were then collected by centrifugation, treated for $10 \mathrm{~min}$ with hypotonic $\mathrm{KCl}$ solution $75 \mathrm{mM}$ (Sigma-Aldrich, USA), fixed in methanol: glacial acetic acid (3:1 v/v), and processed for chromosomal aberration analysis. (Pantelias and Ter- zoudi 2011). Standard procedures were also followed for chromosome preparation and staining. Metaphase spreads were stained with 5\% Giemsa solution (Merck, Germany) for $10 \mathrm{~min}$.

Chromosomal damage was visualized and quantified as chromatid breaks (terminal deletions) and chromatid gaps (achromatic lesions) in cells at metaphase (IAEA 2011). For each subject, 800 cells ( 400 from exposed and 400 from control samples) from 8 different slides ( 100 cells from each slide) identically processed, were scored by light microscopy for chromatid aberrations (gaps and breaks). Gaps were scored only when extended across (occupied) the full chromatid width. An aberration was considered as "break" when the gap width was equal or greater than the chromatid width. Light microscopy was coupled with an image analysis system (Ikaros MetaSystems, Germany) to facilitate scoring. The exposure-induced yield of chromatid aberrations in exposed samples, and the corresponding yield in control samples were blindly scored. Mean values of total number of aberrations (gaps and breaks) per cell and Standard deviation (SD) in exposed and control samples were calculated for each individual.

\section{MT EMF exposure system}

Exposures were performed by a UMTS (3G) commercially available mobile phone handset in order to test the effects of real-life exposures experienced daily by billions of MT users around the world. UMTS employs the "Wideband Code Division Multiple Access" (W-CDMA) code to offer greater spectral efficiency and bandwidth to mobile network operators. All modern digital MT signals, combine both high (RF) and low (ELF) frequencies. The CDMA system assigns a special electronic code to each call allowing the entire frequency band to be occupied simultaneously. Thus, the RF frequency of the carrier signal varies continuously during a conversation. Moreover, the intensity of the EMFemission varies significantly and unpredictably during an active call both in the RF and ELF bands (Panagopoulos et al. 2007, 2010, 2015a; Panagopoulos 2011, 2017, 2019). UMTS (3G) signals use RF carrier frequencies between 1900 and $2200 \mathrm{MHz}$, and ELF pulsing mainly at $100 \mathrm{~Hz}$ and $1500 \mathrm{~Hz}$ (Holma and Toskala 2004; Curwen and Whalley 2008; Health Protection Agency 2012). SAR value of the handset for the human head according to the manufacturer is $0.66 \mathrm{~W} / \mathrm{kg}$.

The RF radiation intensity, emitted by the handset during the exposures was measured at $1 \mathrm{~cm}$ distance from the handset by a Cornet ED85EXpluss RF meter (Cornet Microsystems Inc., USA), and a Spectran HF-4040V3 spectrum analyzer (Aaronia AG, Germany), both with a near-field antenna. The ELF electric and magnetic field intensities (ELF-E and ELF-B) emitted by the handset were measured at $1 \mathrm{~cm}$ distance by a Spectran NF-1010E (Aaronia 
AG, Germany) spectrum analyzer. Representative average power density (from five representative instant measurements excluding background) in the RF band \pm SD was $92 \pm$ $27 \mu \mathrm{W} / \mathrm{cm}^{2}$. The carrier frequency was variable $\sim 1920-1960$ $\mathrm{MHz}$ during the exposures. Representative average ELF-E and ELF-B (from five representative instant measurements excluding background) $\pm \mathrm{SD}$ at $100 \mathrm{~Hz}$ was $12 \pm 4.2 \mathrm{~V} / \mathrm{m}$, and $0.9 \pm 0.4 \mathrm{mG}$, respectively. Corresponding average ELF$\mathrm{E}$ and ELF-B (from five instant measurements excluding background) $\pm \mathrm{SD}$ at $1500 \mathrm{~Hz}$ was $8 \pm 4.6 \mathrm{~V} / \mathrm{m}$, and $0.06 \pm$ $0.02 \mathrm{mG}$, respectively. All measurements were carried out separately from the exposures in order to have the measuring devices at exactly the same position with the samples during the exposures. The above measured EMF/radiation intensity values are representative for UMTS mobile phone EMFs during "talk" mode, and are well within the current exposure limits (ICNIRP 1998, 2010).

\section{EMF exposure procedure}

Whole blood samples were exposed within the $30 \mathrm{ml}$ flasks to modulated emission ("talk" signal) from a commercially available UMTS (3G) mobile phone handset during an active phone-call for $15 \mathrm{~min}$ at $1 \mathrm{~cm}$ distance from the proximal flask wall. This took place in the exposure room so that the controls (in the culture room) would not be exposed. After the exposed samples were back in the culture room, the control (sham-exposed) samples were also transferred in the exposure room for the same time ( $15 \mathrm{~min}$ ) at the same location as the exposed samples without being exposed to the MT EMF. This was done because the background ELF-E and ELF-B and the light conditions in the two rooms were different.

The temperature in the two rooms was the same during the procedures/exposures and was kept at $22 \pm 1^{\circ} \mathrm{C}$. In both rooms the RF background was below $0.01 \mu \mathrm{W} / \mathrm{cm}^{2}$. In the exposure room the ELF-E background was $\sim 2 \mathrm{~V} / \mathrm{m}$, and the ELF-B background $\sim 0.3 \mathrm{mG}(0.03 \mu \mathrm{T})$. In the culture room the corresponding ELF background fields were higher ( $\mathrm{E}$ $10 \mathrm{~V} / \mathrm{m}, \mathrm{B} \sim 1 \mathrm{mG}$ ). [This plus the different light conditions in the two rooms were the reasons why the control blood samples were "sham-exposed" (transferred for the same time in the exposure room)]. Temperature increases within the blood samples during the $15 \mathrm{~min}$ exposures did not exceed $0.1^{\circ} \mathrm{C}$ as measured within an identical culture and flask by a HANNA CheckTemp 1 calibrated electronic thermometer (USA). The handset was fully charged and had full signal reception during the exposures.

\section{Statistical analysis}

Results were statistically analyzed by application of the Student's $t$-test for unequal variances (Microsoft Excel program) between exposed and control groups for each individual. The $p$-values $\leq 0.05$ for the probability that differences between groups are due to random variations were accepted as statistically significant.

\section{Results}

Results from experiments with the 6 healthy subjects (No. 1-6) with 800 metaphases scored from each one (400 from exposed and 400 from control blood samples) are listed in Table 1 and represented graphically in Figure 1. A single 15-min exposure by the UMTS mobile phone during a phone call in "talk" mode at $1 \mathrm{~cm}$ distance, increased the number of chromosomal aberrations (total number of gaps and breaks) by 100-275\% in regards to the unexposed/

Table 1. Chromosomal Aberrations in the Control and Exposed blood samples of 6 subjects, induced by UMTS MT EMF

\begin{tabular}{|c|c|c|c|c|c|c|c|c|c|}
\hline $\begin{array}{l}\text { Subject } \\
\text { No. }\end{array}$ & Age & Sex & Groups & $\begin{array}{l}\text { Gaps in } \\
400 \text { cells }\end{array}$ & $\begin{array}{l}\text { Breaks in } \\
400 \text { cells }\end{array}$ & $\begin{array}{l}\text { Total Aberr. } \\
\text { in } 400 \text { cells }\end{array}$ & $\begin{array}{c}\text { Mean Total Aberr. } \\
\text { per cell } \pm \text { SD }\end{array}$ & $\begin{array}{l}\text { Deviation from } \\
\text { Control }\end{array}$ & $\begin{array}{l}p \text {-value between Exposed } \\
\text { and Control groups }\end{array}$ \\
\hline \multirow{2}{*}{1} & \multirow{2}{*}{42} & \multirow{2}{*}{ Male } & Control & 30 & 5 & 35 & $0.09 \pm 0.03$ & & \\
\hline & & & Exposed & 84 & 17 & 101 & $0.25 \pm 0.08$ & $+178 \%$ & $<0.02$ \\
\hline \multirow{2}{*}{2} & \multirow{2}{*}{33} & \multirow{2}{*}{ Female } & Control & 37 & 7 & 44 & $0.11 \pm 0.04$ & & \\
\hline & & & Exposed & 70 & 19 & 89 & $0.22 \pm 0.06$ & $+100 \%$ & $<0.03$ \\
\hline \multirow{2}{*}{3} & \multirow{2}{*}{28} & \multirow{2}{*}{ Male } & Control & 28 & 9 & 37 & $0.09 \pm 0.03$ & & \\
\hline & & & Exposed & 63 & 15 & 78 & $0.19 \pm 0.04$ & $+111 \%$ & $<0.02$ \\
\hline \multirow{2}{*}{4} & \multirow{2}{*}{40} & \multirow{2}{*}{ Male } & Control & 43 & 15 & 58 & $0.14 \pm 0.04$ & & \\
\hline & & & Exposed & 102 & 26 & 128 & $0.32 \pm 0.09$ & $+129 \%$ & $<0.03$ \\
\hline \multirow{2}{*}{5} & \multirow{2}{*}{35} & \multirow{2}{*}{ Female } & Control & 42 & 2 & 44 & $0.11 \pm 0.01$ & & \\
\hline & & & Exposed & 82 & 12 & 94 & $0.23 \pm 0.03$ & $+109 \%$ & $<0.01$ \\
\hline \multirow{2}{*}{6} & \multirow{2}{*}{30} & \multirow{2}{*}{ Male } & Control & 15 & 2 & 17 & $0.04 \pm 0.01$ & & \\
\hline & & & Exposed & 56 & 5 & 61 & $0.15 \pm 0.04$ & $+275 \%$ & $<0.01$ \\
\hline
\end{tabular}

Aberr.: Aberrations 


\section{Chromosome Damage Induced by UMTS MT EMF}

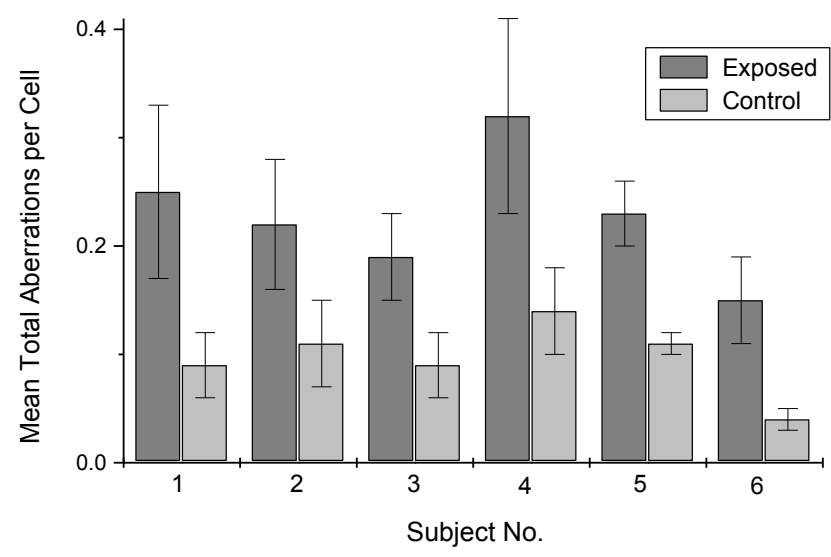

Figure 1. Mean total number of aberrations (gaps and breaks) per cell \pm SD, in 400 exposed and 400 control cells (peripheral blood lymphocytes), for each one of the 6 individuals (No. 1-6), after a single 15 min exposure to UMTS MT EMF.

control samples. The results were in all cases statistically significant $(p<0.03)$ (Table 1$)$.

In Figure 2A a metaphase of a control blood sample is shown from individual No. 4 (male). This is a representative picture of a metaphase with all 46 chromosomes intact. Fig. 2B shows a metaphase of a blood sample of the same individual, exposed to UMTS MT EMF with one chromatid achromatic lesion (gap). In Figure 3A a metaphase of a control blood sample of individual No. 2 (female) is shown with all 46 chromosomes intact. Figure 3B shows a metaphase of a blood sample of the same individual (No. 2), exposed to UMTS MT EMF with two chromatid achromatic lesions (gaps). In Figure 4A a metaphase of a control blood sample of individual No. 6 (male) is shown with all 46 chromosomes intact. Figure $4 \mathrm{~B}$ shows a metaphase of a blood sample of the same individual (No. 6) exposed to UMTS MT EMF with one chromatid achromatic lesion (gap). Figure 4C shows another metaphase of an exposed blood sample of the same individual (No. 6) with one terminal deletion (break) in which the fragment is found away from its chromatid.

Each individual exhibited a different sensitivity to the MT EMF exposure. The differential sensitivity was recorded not only in regard to the MT EMF exposure, but also in the unexposed (control) blood samples. The mean number of total aberrations per cell in the control samples varied from 0.04 to 0.14 , and in the exposed samples from 0.15 to 0.32 , between the 6 different healthy individuals. Subjects with less aberrations in their control samples exhibited higher sensitivity to the MT EMF-exposure (Table 1).

The MT EMF exposure induced mainly gaps, but also breaks in smaller percentages. The number of induced gaps was 4-7 times greater than the number of induced breaks in all individuals. The separate numbers of either gaps or breaks (alike with the total number of aberrations) were significantly increased in regards to the corresponding numbers in the control samples of each individual (Table 1, Fig. 1).

\section{Discussion}

In the present study it is shown that a single 15 min exposure of human blood samples to an active 3G (UMTS) mobile phone in "talk" mode at $1 \mathrm{~cm}$ distance from the handset induces chromosomal aberrations in a significant degree (from $100 \%$ up to $275 \%$ in regards to the control samples), depending on the sensitivity of each individual. The differential sensitivity between different healthy individuals found in the present study in regard to microwave EMF exposure has also been reported before in regard to ionizing (gamma) radiation exposure as assessed with the same $\left(G_{2}\right)$ assay (Pantelias and Terzoudi 2011).
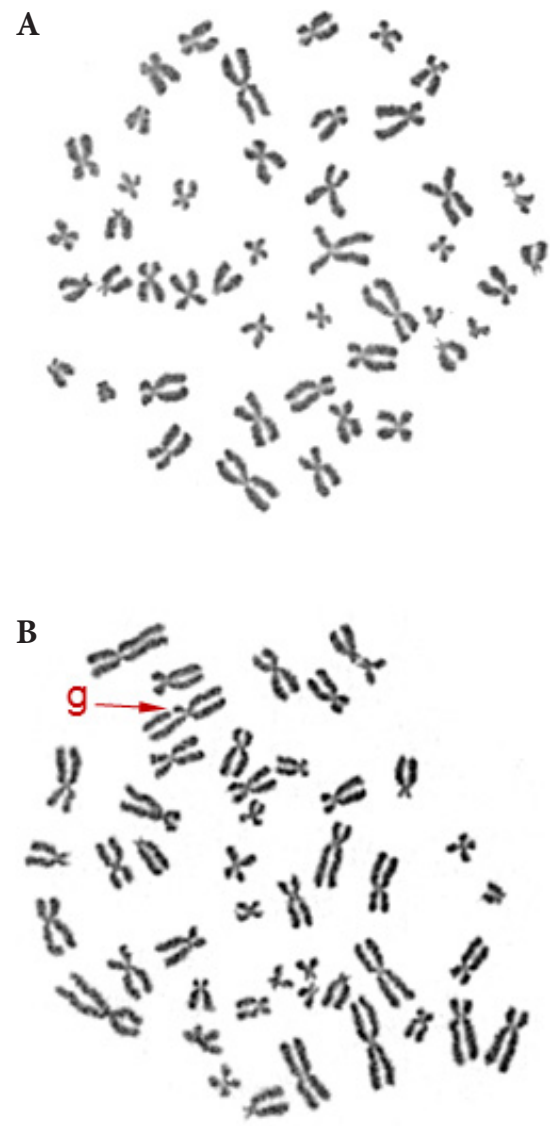

Figure 2. A. Metaphase of Control blood sample from individual No. 4 (male). All 46 chromosomes are intact. B. Metaphase of Exposed blood sample to UMTS MT EMF from individual No. 4 (male) with 1 achromatic lesion - gap (g). 
In previous studies of my group regarding exposure of fruit flies to $2 \mathrm{G}$ (GSM) mobile phone radiation, it was found that this EMF/radiation induced extensive DNA damage in the gametes leading to cell death and to reproductive decline (Panagopoulos et al. 2007, 2010; Chavdoula et al. 2010; Panagopoulos 2012). In addition, it is well documented that DNA damage is converted into chromosomal aberrations during mitosis (Terzoudi and Pantelias 2006; Pantelias and Terzoudi 2010; Terzoudi et al. 2011). Thus the recorded chromosomal damage in human peripheral blood lymphocytes in my present experiments induced by the UMTS (3G) MT EMF is probably due to DNA damage caused by this field/radiation, and could not be repaired or arrested at the checkpoint
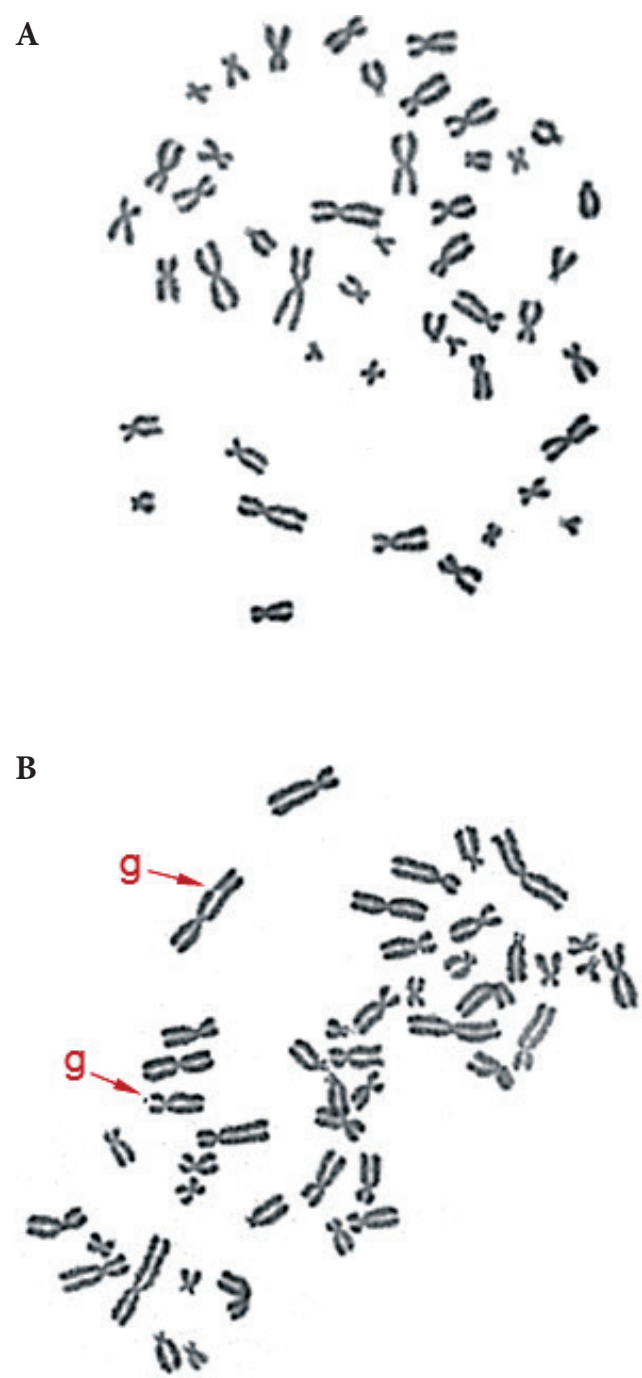

Figure 3. A. Metaphase of Control blood sample from individual No. 2 (female), with all 46 chromosomes intact. B. Metaphase of Exposed blood sample to UMTS MT EMF from individual No. 2 (female) with 2 achromatic lesions - gaps (g). during the $G_{2}$ phase since many cells had probably already passed the checkpoint during the exposure. This should be further investigated by future experiments.

The results of the present experiments are in agreement with the previous results of my group (Panagopoulos et al. 2007, 2010; Chavdoula et al. 2010; Panagopoulos 2012) and once more show that digital MT microwave EMFs are very genotoxic/bioactive, able to induce DNA damage and consequent chromosomal aberrations in the human cells as well as in other animals. This should be anticipated since cells are essentially the same in all animals, and all biological/health effects are initiated at the cellular level (Panagopoulos 2019).

The recorded effect is non-thermal since it was not accompanied by any significant temperature increase of the exposed blood samples. The $0.1^{\circ} \mathrm{C}$ temperature increase during the 15 min exposures is well tolerated by the blood cells. It is known that blood samples are ideally maintained at temperatures between 18 and $24^{\circ} \mathrm{C}$ (IAEA 2011). As we described, the blood samples were treated and exposed at room temperature around $22^{\circ} \mathrm{C}$, and also cultured at $37^{\circ} \mathrm{C}$. These temperatures are standard in different laboratories working with human peripheral blood lymphocytes (IAEA 2011). Since the $15^{\circ} \mathrm{C}$ temperature difference between culturing and treatment had no significant effect on the blood lymphocytes of the control samples we can certainly accept that the $0.1^{\circ} \mathrm{C}$ temperature increase during the $15 \mathrm{~min}$ exposures is totally insignificant to have any effect on the quality of the samples. In my previous experiments with the GSM MT EMFs no temperature increases were detected during exposures up to $21 \mathrm{~min}$ (Panagopoulos 2011, 2017). The $0.1^{\circ} \mathrm{C}$ temperature increase detected in the present experiments with UMTS MT EMFs is probably due to the higher carrier frequency of UMTS $(\sim 1920-1960 \mathrm{MHz})$ than that of GSM ( 900 or $\sim 1800 \mathrm{MHz}$ ), since the amount of microwave power absorbed by exposed materials and converted to heat is proportional to the (carrier) microwave frequency (Clark et al. 2000). The newest types of MT employ significantly higher carrier frequencies, $4 \mathrm{G}$ up to $2.6 \mathrm{GHz}$, and the upcoming $5 \mathrm{G}$ up to $100 \mathrm{GHz}$. Thus, especially $5 \mathrm{G}$ is expected to induce significant thermal effects in addition to the non-thermal ones which may not be tolerated by the human/animal body (Singh et al. 2017; Neufeld and Kuster 2018). This may represent a great danger for public health, which the health authorities should carefully investigate before allowing $5 \mathrm{G}$ installation.

The present experiments show that the $G_{2}$ assay is sensitive enough to detect effects on DNA from milder agents than ionizing radiation, such as EMFs of modern mobile telecommunications and possibly other types of man-made EMFs as well. The main type of aberrations found were chromatid gaps (achromatic lesions). In all blood samples from all six individuals the number of induced chromatid gaps was significantly (4-7 times) greater than the number 
of induced chromatid breaks. Both gaps and breaks are considered to be aberrations of the same nature. More specifically, it is established that "gaps and breaks are different manifestations of the same events" and gaps are actually incomplete breaks (Brecher 1977). It is obvious though that breaks (terminal deletions) are more intense damages and easier to be recognized through an optical microscope than gaps (achromatic lesions). A terminal deletion (break) will release its fragment at anaphase, while the gap probably will not (Conger 1967). Therefore when the $G_{2}$ assay is applied to test EMF-bioactivity, the examination should be focused in detecting minor aberrations (chromatid gaps) in addition to the major ones (chromatid breaks etc.). The chromosomes should be carefully examined in order to detect the smaller damages (gaps) which in such a case are the majority, in contrast to damages induced by ionizing radiation which are usually more extended and easily observed. In any case, my opinion is that both breaks and gaps should be scored, although counting only the breaks can make the scoring significantly faster. Ignoring the smaller damages (gaps) and observing only the more extended ones (breaks), may be another reason why certain previous studies did not report chromosome aberrations in human blood lymphocytes, in addition to the use of simulated MT signals.

As mentioned in the introduction, previous studies that tested the bioactivity of simulated MT EMFs emitted by generators on human lymphocytes had reported contradicting results (Zeni et al. 2003, 2012; Markova et al. 2005; Belyaev et al. 2005, 2009; Baohong et al. 2005, 2007; Schwarz et al. 2008; Manti et al. 2008; El-Abd and Eltoweissy 2012). We have previously shown that real-life MT EMFs emitted by commercially available mobile phone devices or base antennas/cell towers are far more bioactive than simulated corresponding signals with invariable parameters emitted by generators (Panagopoulos et al. 2015a; Panagopoulos 2017, 2019). It seems to be for the same reason why in some of the previous studies no effects of simulated MT EMFs on human lymphocytes were reported (Schwarz et al. 2008; Zeni et al. 2003, 2012), while in the present study in which a real UMTS exposure was employed, a very intense effect was found (up to $275 \%$ increase in chromatid aberrations in regards to the control samples). An older study on peripheral blood leukocytes from radar-facility workers exposed to radar microwave radiation $1250-1350 \mathrm{MHz}, 10-20000$ $\mu \mathrm{W} / \mathrm{cm}^{2}$, found DNA damage and chromatid breaks almost 3 times higher (300\%) than in control (unexposed) subjects (Garaj-Vrhovac and Orescanin 2009). [Microwave radiation emitted by radars is invariable (as it carries no information) but of much higher intensity (up to 1000 times) close to the antenna compared to mobile phone radiation].

Two recent studies that examined peripheral blood lymphocytes from people residing close to MT base antennas, and thus exposed to real-life MT EMFs, found significantly
A

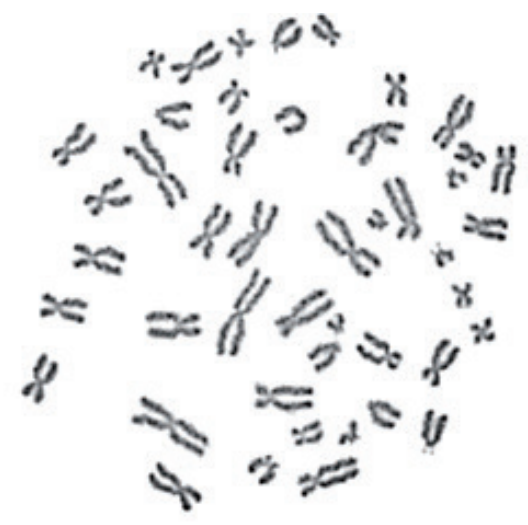

B

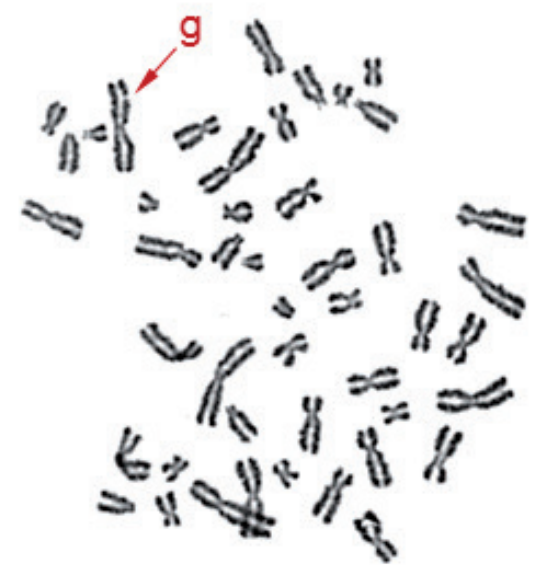

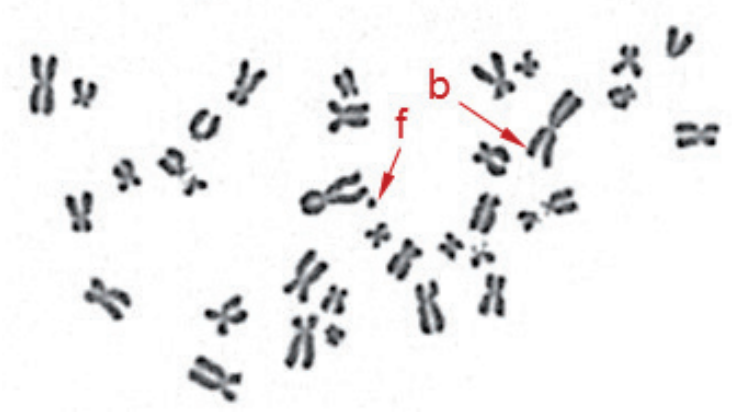

Figure 4. A. Metaphase of Control blood sample from individual No. 6 (male), with all 46 chromosomes intact. B. Metaphase of Exposed blood sample to UMTS MT EMF from individual No. 6 (male) with 1 achromatic lesion - gap (g). C. Metaphase of Exposed blood sample to UMTS MT EMF from individual No. 6 (male) with 1 terminal deletion - break (b). f: fragment. 
higher genetic damage than from people residing further than $300 \mathrm{~m}$ (Gulati et al. 2016; Zothansiama et al. 2017). Another recent study that employed a real UMTS or GSM exposure by an active commercially available mobile phone (D'Silva et al. 2017) also found induction of genotoxic effect in both cases, with the UMTS found to be even more genotoxic than the GSM EMF. These results are in line with the present results as well as with the fact that newer types of MT EMFs (3G, 4G, etc.) transmit increasingly higher amount/ density of variable information (speech, text, images, video, Internet) making the signal increasingly complicated and unpredictably variable each moment. This increasingly higher and unpredictable variability of the newer types of telecommunication EMFs makes them more and more bioactive due to the inability of living organisms to adapt, in contrast to simulated MT EMFs which are invariable and thus absolutely predictable (Panagopoulos et al. 2015a; Panagopoulos 2017, 2019).

The disruption of cell electrochemical balance by manmade (polarized) EMFs through irregular gating of voltagegated ion channels on cell membranes is described by the "ion forced-oscillation mechanism" (Panagopoulos et al. 2000, 2002, 2015b). In turn, it is explained how the disruption of intracellular ionic concentrations may lead to DNA damage by intracellular release of free radicals or hydrolytic enzymes like DNases (Barzilai and Yamamoto 2004; Phillips et al. 2009; Panagopoulos 2011; Pall 2013). This is in line with the attribution of the DNA and chromosome damage to oxidative stress by El-Abd and Eltoweissy (2012). According to the ion forced-oscillation mechanism, the bioactivity of a polarized EMF is proportional to its intensity and inversely proportional to its frequency, meaning that the ELF pulsing and modulation of the MT EMFs seem to be responsible for their intense bioactivity and not the carrier (RF) frequency which is several orders of magnitude higher than ELF. This is supported by significant experimental evidence ( $\mathrm{Pa}$ nagopoulos 2019). Thus, reports that MT EMF-bioactivity depends on carrier frequency (Markova et al. 2005) seem unlikely according to this mechanism and the corresponding experimental evidence.

The present study - in line with previous studies of my group - indicates that people should necessarily reduce exposures to MT EMFs as much as possible by making prudent use of this technology and reduce drastically the number and duration of calls, make use of wired headsets (especially air-tube headsets) or the loudspeaker during calls and keep the device at the greatest possible distance from the body, not carry the mobile phones on their bodies while they are turned on, keep them at the greatest possible distance during the day, and switch them off during sleep. An avoidance strategy is the key for protection against the tremendously increased levels of man-made EMF exposures rather than metal shielding which is accused for internal desynchro- nization. This is a severe medical syndrome in which the circadian rhythms of an individual (activity, temperature sleep/waking cycles, body secretions, etc.) start deviating from their normal $24 \mathrm{~h}$-periodicity. Long-term persistence of this phenomenon is connected to a variety of health problems such as depression, sleep disorders, impulsivity, mania, metabolic syndrome, cardiovascular disease, and increased cancer risk (Panagopoulos and Chrousos 2019). Finally, the responsible public health authorities should take into account the results of the present study as well as the results of hundreds of other peer-reviewed published studies (Panagopoulos 2019) and establish much stringer exposure limits than the existing ones.

Acknowledgements. I thank Drs G. Pantelias, G. Terzoudi, M. Karakosta, and V. Hatzi, for showing me the laboratory techniques with the human lymphocytes and particularly the $\mathrm{G}_{2}$ assay. I also thank the laboratory staff members A. Vasilaki and K. Barszczewska for laboratory assistance during the experiments and help with the blind scoring of the samples.

Conflict of interest. The author declares no actual or potential competing financial interests.

\section{References}

Baohong W, Jiliang H, Lifen J, Deqiang L, Wei Z, Jianlin L, Hongping D (2005): Studying the synergistic damage effects induced by $1.8 \mathrm{GHz}$ radiofrequency field radiation (RFR) with four chemical mutagens on human lymphocyte DNA using comet assay in vitro. Mutat Res. 578, 149-157

https://doi.org/10.1016/j.mrfmmm.2005.05.001

Baohong W, Lifen J, Lanjuan L, Jianlin L, Deqiang L, Wei Z, Jiliang $H$ (2007): Evaluating the combinative effects on human lymphocyte DNA damage induced by ultraviolet ray $\mathrm{C}$ plus $1.8 \mathrm{GHz}$ microwaves using comet assay in vitro. Toxicology 232, 311-316 https://doi.org/10.1016/j.tox.2007.01.019

Barzilai A, Yamamoto K (2004): DNA damage responses to oxidative stress. (Review). DNA Repair 3, 1109-1115 https://doi.org/10.1016/j.dnarep.2004.03.002

Belyaev IY, Hillert L, Protopopova M, Tamm C, Malmgren LO, Persson BR, Selivanova G, Harms-Ringdahl M (2005): 915 $\mathrm{MHz}$ microwaves and $50 \mathrm{~Hz}$ magnetic field affect chromatin conformation and 53BP1 foci in human lymphocytes from hypersensitive and healthy persons. Bioelectromagnetics 26, 173-184 https://doi.org/10.1002/bem.20103

Belyaev IY, Markovà E, Hillert L, Malmgren LOG, Persson BRR (2009): Microwaves from UMTS/GSM mobile phones induce long-lasting inhibition of 53BP $1 / \gamma-\mathrm{H} 2 \mathrm{AX}$ DNA repair foci in human lymphocytes. Bioelectromagnetics 30, 129-141 https://doi.org/10.1002/bem.20445

Brecher S (1977): Ultra-structural observations of x-ray induced chromatid gaps. Mutat. Res. 42, 249-268 
https://doi.org/10.1016/S0027-5107(77)80028-6

Chavdoula ED, Panagopoulos DJ, Margaritis LH (2010): Comparison of biological effects between continuous and intermittent exposure to GSM-900 MHz mobile phone radiation. Detection of apoptotic cell death features. Mutat. Res. 700, 51-61 https://doi.org/10.1016/j.mrgentox.2010.05.008

Clark DE, Folz DC, West JK (2000): Processing materials with microwave energy. Mater. Sci. Eng. 287, 153-158 https://doi.org/10.1016/S0921-5093(00)00768-1

Conger AD (1967): Real chromatid deletions versus gaps. Mutat. Res. 4, 449-459 https://doi.org/10.1016/0027-5107(67)90007-3

Curwen P, Whalley J (2008): Mobile communications in the 21st century. In: Mobile Telephones: Networks, Applications and Performance. (Eds. Harper AC and Buress RV), pp. 29-75, Nova Science Publishers

D'Silva MH, Swer RT, Anbalagan J, Rajesh B (2017): Effect of radiofrequency radiation emitted from $2 \mathrm{G}$ and $3 \mathrm{G}$ cell phone on developing liver of chick embryo - a comparative study. J. Clin. Diagn. Res. 11, 5-9 https://doi.org/10.7860/JCDR/2017/26360.10275

El-Abd SF, Eltoweissy MY (2012): Cytogenetic alterations in human lymphocyte culture following exposure to radiofrequency field of mobile phone. J. App. Pharm. Sci. 2, 16-20

Falcioni L, Bua L, Tibaldi E, Lauriola M, De Angelis L, Gnudi F, Mandrioli D, Manservigi M, Manservisi F, Manzoli I, et al. (2018): Report of final results regarding brain and heart tumors in Sprague-Dawley rats exposed from prenatal life until natural death to mobile phone radiofrequency field representative of a 1.8GHz GSM base station environmental emission. Environ. Res. 165, 496-503 https://doi.org/10.1016/j.envres.2018.01.037

Garaj-Vrhovac V, Orescanin V (2009): Assessment of DNA sensitivity in peripheral blood leukocytes after occupational exposure to microwave radiation: the alkaline comet assay and chromatid breakage assay. Cell Biol. Toxicol. 25, 33-43 https://doi.org/10.1007/s10565-008-9060-3

Gulati S, Yadav A, Kumar N, Kanupriya, Aggarwal NK, Kumar R, Gupta R (2016): Effect of GSTM1 and GSTT1 polymorphisms on genetic damage in humans populations exposed to radiation from mobile towers. Arch. Environ. Contam. Toxicol. 70, 615-625 https://doi.org/10.1007/s00244-015-0195-y

Health Protection Agency (2012): Health Effects from Radiofrequency Electromagnetic Fields. ISBN 978-0-85951-714-0

Holma H, Toskala A (2004): WCDMA for UMTS, Radio Access for Third Generation Mobile Communications. John Wiley \& Sons Inc.

IAEA (2011): International Atomic Energy Agency. Cytogenetic Dosimetry: Applications in Preparedness for and Response to Radiation Emergencies

IARC (2002): Non-Ionizing Radiation. Part 1: Static and Extremely Low-Frequency (ELF) Electric and Magnetic Fields, Vol. 80, World Health Organization

IARC (2013): Non-Ionizing Radiation. Part 2: Radiofrequency Electromagnetic Fields, Vol. 102, World Health Organization

ICNIRP (1998): Guidelines for limiting exposure to time-varying electric, magnetic and electromagnetic fields (up to $300 \mathrm{GHz}$ ). Health Phys. 74, 494-522
ICNIRP (2010): Guidelines for limiting exposure to time-varying electric and magnetic fields $(1 \mathrm{~Hz}-100 \mathrm{kHz})$. Health Phys. 99, 818-836

https://doi.org/10.1097/01.HP.0000391657.33635.13

Manti L, Braselmann H, Calabrese ML, Massa R, Pugliese M, Scampoli P, Sicignano G, Grossi G (2008): Effects of modulated microwave radiation at cellular telephone frequency (1.95 $\mathrm{GHz}$ ) on X-ray-induced chromosome aberrations in human lymphocytes In Vitro. Radiat. Res. 169, 575-583 https://doi.org/10.1667/RR1044.1

Markova E, Hillert L, Malmgren L, Persson BR, Belyaev IY (2005): Microwaves from GSM mobile telephones affect 53BP1 and gamma-H2AX foci in human lymphocytes from hypersensitive and healthy persons. Environ. Health Perspect. 113, 1172-1177 https://doi.org/10.1289/ehp.7561

Neufeld E, Kuster N (2018): Systematic derivation of safety limits for time-varying $5 \mathrm{G}$ radiofrequency exposure based on analytical models and thermal dose. Health Physics. 115, 705-711 https://doi.org/10.1097/HP.0000000000000930

NTP (2018): National Toxicology Program: Toxicology and Carcinogenesis Studies in Hsd: Sprague Dawley SD Rats Exposed to Whole-body Radio Frequency Radiation at a Frequency (900 MHz) and Modulations (GSM and CDMA) Used by Cell Phones. NTP TR 595, Department of Health and Human Services, USA

Pall ML (2013): Electromagnetic fields act via activation of voltagegated calcium channels to produce beneficial or adverse effects. J. Cell. Mol. Med. 17, 958-965 https://doi.org/10.1111/jcmm.12088

Panagopoulos DJ, Messini N, Karabarbounis A, Filippetis AL, Margaritis LH (2000): A mechanism for action of oscillating electric fields on cells. Biochem. Biophys. Res. Commun. 272, 634-640 https://doi.org/10.1006/bbrc.2000.2746

Panagopoulos DJ, Karabarbounis A, Margaritis LH (2002): Mechanism for action of electromagnetic fields on cells. Biochem. Biophys. Res. Commun. 298, 95-102

https://doi.org/10.1016/S0006-291X(02)02393-8

Panagopoulos DJ, Chavdoula ED, Nezis IP, Margaritis LH (2007): Cell death induced by GSM 900MHz and DCS $1800 \mathrm{MHz}$ mobile telephony radiation. Mutat. Res. 626, 69-78 https://doi.org/10.1016/j.mrgentox.2006.08.008

Panagopoulos DJ, Chavdoula ED, Margaritis LH (2010): Bioeffects of mobile telephony radiation in relation to its intensity or distance from the antenna. Int. J. Radiat. Biol. 86, 345-357 https://doi.org/10.3109/09553000903567961

Panagopoulos DJ (2011): Analyzing the health impacts of modern telecommunications microwaves. In: Advances in Medicine and Biology (Ed. Berhardt LV), Vol. 17, Nova Science Publishers, Inc., New York, USA

Panagopoulos DJ (2012): Effect of microwave exposure on the ovarian development of drosophila melanogaster. Cell Biochem. Biophys. 63, 121-132 https://doi.org/10.1007/s12013-012-9347-0

Panagopoulos DJ, Johansson O, Carlo GL (2015a): Real versus simulated mobile phone exposures in experimental studies. Biomed. Res. Int. 2015, 607053 https://doi.org/10.1155/2015/607053 
Panagopoulos DJ, Johansson O, Carlo GL (2015b): Polarization: A key difference between man-made and natural electromagnetic fields, in regard to biological activity. Scientific Reports 5, 14914 https://doi.org/10.1038/srep14914

Panagopoulos DJ (2017): Mobile telephony radiation effects on insect ovarian cells. The necessity for real exposures bioactivity assessment. The key role of polarization, and the ion forcedoscillation mechanism. In: Microwave Effects on DNA and Proteins (Ed. Geddes CD), Springer https://doi.org/10.1007/978-3-319-50289-2_1

Panagopoulos DJ, Chrousos GP (2019): Shielding methods and products against man-made electromagnetic fields: protection versus risk. Sci. Total Environ. 667, 255-262

https://doi.org/10.1016/j.scitotenv.2019.02.344

Panagopoulos DJ (2019): Comparing DNA damage induced by mobile telephony and other types of man-made electromagnetic fields. Mutat. Res. Rev. 781, 53-62 https://doi.org/10.1016/j.mrrev.2019.03.003

Pantelias GE, Terzoudi GI (2010): Functional cell-cycle chromatin conformation changes in the presence of DNA damage result into chromatid breaks. A new insight in the formation of radiation-induced chromosomal aberrations based on the direct observation of interphase chromatin. Mutat. Res. 701, 27-37 https://doi.org/10.1016/j.mrgentox.2010.04.006

Pantelias GE, Terzoudi GI (2011): A standardized G2-assay for the prediction of individual radiosensitivity. Radiotherap. Oncol. 101, 28-34 https://doi.org/10.1016/j.radonc.2011.09.021

Phillips JL, Singh NP, Lai H, (2009): Electromagnetic fields and DNA damage. Pathophysiology 16, 79-88. https://doi.org/10.1016/j.pathophys.2008.11.005

Schwarz C, Kratochvil E, Pilger A, Kuster N, Adlkofer F, Ruediger HW (2008): Radiofrequency electromagnetic fields (UMTS, $1,950 \mathrm{MHz}$ ) induce genotoxic effects in vitro in human fibroblasts but not in lymphocytes. Int. Arch. Occup. Environ. Health 81, 755-767 https://doi.org/10.1007/s00420-008-0305-5

Singh RK, Bisht D, Prasad RC (2017): Development of 5G mobile network technology and its architecture. Int. J. Recent Trends in Eng. Res. 3, 196-201

https://doi.org/10.23883/IJRTER.2017.3475.VMOF0

Terzoudi GI, Pantelias GE (2006): Cytogenetic methods for biodosimetry and risk individualization after exposure to ionizing radiation. Radiat. Prot. Dosimetry 122, 513-520 https://doi.org/10.1093/rpd/ncl509

Terzoudi GI, Hatzi VI, Donta-Bakoyianni C, Pantelias GE (2011): Chromatin dynamics during cell cycle mediate conversion of DNA damage into chromatid breaks and affect formation of chromosomal aberrations: biological and clinical significance. Mutat. Res. 711, 174-186 https://doi.org/10.1016/j.mrfmmm.2010.12.011

Zeni O, Chiavoni AS, Sannino A, Antolini A, Forigo D, Bersani F, Scarfi MR (2003): Lack of genotoxic effects (micronucleus induction) in human lymphocytes exposed in vitro to $900 \mathrm{MHz}$ electromagnetic fields. Radiat. Res. 160, 152-158 https://doi.org/10.1667/RR3014

Zeni O, Sannino A, Romeo S, Massa R, Sarti M, Reddy AB, Prihoda TJ, Vijaya-laxmi, Scarfi MR (2012): Induction of an adaptive response in human blood lymphocytes exposed to radiofrequency fields: influence of the universal mobile telecommunication system (UMTS) signal and the specific absorption rate. Mutat Res. 747, 29-35 https://doi.org/10.1016/j.mrgentox.2012.03.013

Zothansiama, Zosangzuali M, Lalramdinpuii M, Jagetia GC (2017): Impact of radiofrequency radiation on DNA damage and antioxidants in peripheral blood lymphocytes of humans residing in the vicinity of mobile phone base stations. Electromagn. Biol. Med. 36, 295-305 https://doi.org/10.1080/15368378.2017.1350584

Received: June 28, 2019

Final version accepted: August 8, 2019

First published online: September 13, 2019 